\title{
EL PAPEL DE LA HISTORIA Y DE LAS PRÁCTICAS CIENTÍFICAS EN LA EDUCACIÓN
}

\author{
Sergio F. MARTINEZ \\ Instituto de Investigaciones Filosóficas, UNAM
}

Una ojeada a las teorías de la educación que han sido propuestas a lo largo de la historia de la filosofía muestra que nuestro concepto de educación ha estado siempre influido por las diferentes maneras en las que entendemos qué es y qué no es conocimiento. Es mas, muchas teorías clásicas del conocimiento se han concebido simultáneamente como gúas a seguirse para reformar la educación. Ya en los primeros diálogos platónicos se sugiere que educar consiste en promover la investigación de la base de nuestras opiniones con miras a elucidar las interconexiones entre ellas. Esta elucidación nos haría capaces de generar juicios razonados acerca de conflictos entre creencias y prácticas. El ideal de la epistemología clásica que se desarrolla sobre todo en el siglo XVII, según el cual la epistemología debe de buscar el balance entre terapia y evaluación epistemológicas llevaba de manera natural a considerar indispensable la consideración de las implicaciones críticas para la pedagogía de una teoría filosófica acerca de la naturaleza del conocimiento. Es en el marco de este ideal que Descartes y Leibniz promueven una profunda reconsideración en los siglos XVI y XVII del papel de las matemáticas en la educación, y que Locke insiste en que no hay verdades morales innatas, y de que así como todo el conocimiento proviene de la experiencia toda virtud proviene de la educación.

Un supuesto muy antiguo que ha jugado un papel central en muchas teorías de la educación es que el conocimiento se transmite de la cabeza del profesor a la del alumno. Bajo este supuesto la educación consiste en una transferencia de conocimiento por medio del lenguaje (ya sea oral o escrito). El lenguaje sirve como un sistema de signos en el cual el maestro codifica su mensaje que el pupilo que conoce el lenguaje puede decodificar y llegar por lo tanto 
a entender el mensaje. La idea ha sido muy popular en el siglo XX sobre todo por la identificación común que se hace entre información y conocimiento. Es también apoyada por la concepción usual del conocimiento científico como conocimiento de teorías, que a su vez es una versión de la idea que el conocimiento es preeminentemente proposicional, esto es, conocimiento del tipo $S$ sabe que $\mathrm{p}$, donde $S$ es un sujeto y $\mathrm{p}$ una proposición.

Este modelo del conocimiento como transferencia ya fue criticada por San Agustín en el diálogo El Maestro. San Agustín argumenta que es posible aprender cosas sin necesidad de signos intermediarios, y que muchas veces las cosas mas importantes se aprenden así. En relación a las cosas que se perciben con la mente San Agustín piensa que es Dios quién nos las puede hacer manifiestas directamente a la mente. Muy pocos de nosotros estaríamos de acuerdo en formular una alternativa al modelo de la transferencia de esta manera, pero la crítica a este modelo sigue siendo pertinente. Es más, los estudios sobre la ciencia de los últimos años han mostrado que no es posible entender la naturaleza del conocimiento cientifico si nos restringimos a entender el conocimiento como conocimiento teórico, y por lo tanto acorde con alguna versión del modelo de la transferencia. En este trabajo no voy a elaborar una crítica a esta manera de entender el conocimiento, mas bien voy a dar por sentado que dicha crítica es atendible y que una teoría del conocimiento que permita un equilibrio entre los aspectos evaluativos y los terapéuticos debe de tomarse muy en serio la manera como el conocimiento se articula en prácticas y tradiciones ${ }^{1}$. Partiendo de este supuesto exploraré algunas de las implicaciones que tiene el abandono del modelo de la transferencia (término que utilizaremos para referirnos a esta vieja idea que el conocimiento es conocimiento de teorias que se transfiere de persona a persona por medio de algún sistema de información basada en signos lingüísticos) para propuestas acerca de la importancia que tiene el estudio de la ciencia en la educación.

Hay muchas maneras en las que puede incorporarse un estudio de la ciencia en programas de estudio de los diversos niveles educativos. Hoy día es

1 En un sentido importante la articulación en prácticas y tradiciones es un tema central de toda una serie de trabajos en la historia y la sociología de la ciencia, vease por ejemplo el libro compilado por Pickering Science as Practice and Culture, Chicago 1992. Las implicaciones para la filosofia de la ciencia las examino en Filosoffa de las Prácricas Científicas, en prensa, UNAM. Algunas consideraciones críticas del modelo de la transferencia para teorías de la educación las examino en "Autoridad y Conocimiento", Theoria ... 
posible encontrarse con cursos como "Historia de la Ciencia", "Ciencia Tecnología y Sociedad", "Etica de la Ciencia" o "Ciencia y Género" en los programas de estudio de muchas universidades y algunas escuelas preparatorias. No hay sin embargo prácticamente literatura que trate de examinar críticamente todas estas maneras de incorporar la ciencia en la educación. ¿Debemos de ver esas diferentes propuestas de enfocar el estudio de la ciencia fuera de su estudio profesional meramente como alternativas que responden a diferentes tipos de intereses ideológicos o epistemológicos, o debemos de ver esos diferentes tipos de cursos como opciones igualmente válidas y útiles para promover un entendimiento de la naturaleza y posibilidades de la ciencia como conocimiento, si bien desde diferentes perspectivas?

Creo que la respuesta a esta pregunta va a depender (entre cosas) de la manera como entendamos la naturaleza del conocimiento. Si pensamos que el conocimiento es conocimiento teórico entonces vamos a tender a pensar que la historia de la ciencia es la historia del descubrimiento, aceptación y rechazo de teorías. Así entendida la historia de la ciencia puede ayudarnos a entender la naturaleza de la ciencia a través de un estudio de ejemplos de cómo las teorías han ido cambiando, pero otro tipo de cursos difícilmente van a lograr ese cometido. Cursos de Ciencia Tecnología y Sociedad, por ejemplo, en todo caso serían útiles para que los estudiantes tomen nota de las posibles aplicaciones del conocimiento científico y su uso como parte de una cierta ideología, pero no ayudarían en mucho a entender el contenido de la ciencia, sus aspectos metodológicos y epistemológicos distintivos.

Como veremos, si reconocemos que el conocimiento científico es algo mas que conocimiento de teorfas entonces el estudio social de la ciencia desde diferentes perspectivas puede lograr avances en el entendimiento del contenido (axiológico, y en particular epistémico) de la ciencia, no sólo de sus posibles aplicaciones. Desde esta perspectiva el estudio de la ciencia desde los diferentes tipos de cursos que están empezando a ser populares (historia de la ciencia, ciencia tecnología y sociedad, etc) deben de pensarse como contribuyendo a una perspectiva diferente de la ciencia, a una perspectiva en la que la ciencia es parte de un complejo proceso de la individuación y cultivo de los valores que se considera guían toda indagación racional.

En este trabajo voy a partir de un recuento de algunas de las razones que se han ofrecido como apoyo para estudiar la historia de la ciencia. Quiero hacer ver cómo, una vez que se abandona el modelo de la transferencia, estas razones 
son también razones poderosas para incorporar el estudio de la ciencia en el currículo desde muy variadas perspectivas.

Muchas razones pueden darse (y han sido dadas) para apuntalar la incorporación de la historia de la ciencia en los planes educativos a todos los niveles ${ }^{2}$. Hay por un lado razones que muestran la importancia de que los científicos estudien la historia de la ciencia no meramente como parte de los libros de texto de las materias propias de su estudio profesional, sino como parte central de su preparación profesional. Hay por otro razones a favor de la incorporación de la historia de la ciencia en el nivel medio y superior como parte del núcleo del currículo. Adelante formulamos un breve resumen de ambos tipos de razones. En general la idea es que el estudio de la historia de la ciencia como parte de la educación general, no sólo de la educación de un científico, es importante porque nos sirve de modelo acerca de cómo la ciencia toma forma en el contexto de prácticas particulares. Como veremos, una vez que se reconoce el carácter situado (en prácticas y tradiciones) del conocimiento científico este tipo de argumentos se realza, y las razones a favor de estudiar la historia de la ciencia pueden verse a la vez como argumentos por la importancia que tienen los estudios sociales de la ciencia en el currículo.

II. Las razones por las que se piensa que la enseñanza de la historia de la ciencia puede ser importante en la educación de un científico son muy variadas. Entre las principales están las siguientes:

1) El estudio de la historia de la ciencia ayuda a entender la naturaleza de la ciencia como una empresa cultural compleja, y por lo tanto ayuda a situar la educación propiamente profesional en un contexto cultural más amplio.

2) La historia de la ciencia permite una mejor comprensión de los métodos y los conceptos científicos y la manera como esos métodos y conceptos están asociados con fines y valores que se mantienen relativamente estables a lo largo de grandes períodos de tiempo. La historia de la ciencia muestra que esos fines y valores muchas veces atraviesan fronteras disciplinarias y muchas veces contribuyen a la creación o a la reorganización de disciplinas y al desarrollo de avances tecnológicos.

2 En "History of Science in the Currículum" (capítulo 4 de Science Teaching, Routledge, New York, London 1994) Michael Matthews da un resumen de la controversia respecto a si es o no aconsejable ensefiarle historia de la ciencia a los estudiantes de ciencia. 
3) La historia de la ciencia puede ayudar a ver conexiones entre temas, problemas y disciplinas que por lo general quedan ocultos en los libros de texto que tradicionalmente son la base de la educación de un científico. De esta manera el estudio de la historia de la ciencia puede ser de gran ayuda en entender el carácter inter- o multidisciplinario de los problemas científicos, y por lo tanto puede ser de gran ayuda en fomentar el interés del estudiante en tratar de entender el carácter disciplinario de la ciencia a través de sus problemas, y no al revés. La educación usual de un científico, por su tendencia a presentar una estructura rígida de materias, tiende a fomentar la idea que la ciencia es un cúmulo de información que hay que procesar para tener respuestas científicamente apropiadas a los problemas que se presentan en la profesión, y tiende a olvidarse que es el planteamiento de problemas en un contexto social-tecnológico lo que históricamente ha conformado las disciplinas, y no a la inversa. La historia de la ciencia es además un medio muy útil para mostrar el carácter fluido de las disciplinas y la importancia que tienen los esfuerzos trans-disciplinarios a largo plazo dirigidos a la elucidación de conceptos y problemas.

4) Un enfoque histórico al conocimiento científico ayuda a que la manera de pensar de los estudiantes individuales pueda encajar más rápidamente con las maneras establecidas de plantearse los problemas en una disciplina particular. En la historia de la ciencia muchas veces encontramos presupuestos que son compartidos por los estudiantes, y cuya crítica y abandono están asociados a importantes avances cientificos. Muchas personas que no han sido educadas en la ciencia tienen creencias acerca del comportamiento de los cuerpos físicos que se adecuan mas a teorías de los griegos antiguos que las que se consideran hoy día correctas. Incluso estudiantes que llegan a la universidad tienen ideas sobre la evolución de los organismos biológicos que se asemejan mas a teorías que eran comunes antes de Darwin, que a las teorías que hoy día aceptamos como correctas, a partir del replanteamiento de la biología iniciado por Darwin. La historia de la ciencia puede ayudar al estudiante a localizar esos presupuestos incorrectos y a abandonarlos racionalmente.

Cada una de estas razones puede tener diferentes presupuestos que vale la pena hacer explícitos por lo menos de manera breve. Para un positivista radical (entre los cuales se cuentan muchos científicos) la ciencia es una empresa distinguible por un método de investigación cuya comprensión y seguimiento no requiere de saber historia de nada. Según esta concepción positivista de la ciencia el estudio de la historia de la ciencia puede ayudarnos a apreciar estética o moralmente la ciencia, pero no va a ayudarnos a entender la naturaleza 
de la empresa cientifica. Pero alguien menos positivista puede aceptar que detrás del primer tipo de razón está la idea que así como nadie cuestiona la importancia de estudiar la historia de los diferentes valores e instituciones sociales propios de una sociedad para entender mejor esos instituciones y valores, es también importante estudiar la historia de la ciencia para poder entender mejor su naturaleza. Se asume que la ciencia es parte integral de la cultura y que la justificación del estudio de su historia es análoga a la justificación que puede darse para estudiar la historia de la iglesia católica en México. Esto por supuesto no implica que la ciencia es el mismo tipo de empresa cultural que un movimiento religioso, pero sí genera importantes problemas de interpretación de la empresa cientifica y en particular sugiere fuertemente el fantasma del relativismo. Entrar en estos problemas no es nuestra preocupación actual y aqui simplemente asumimos que este historicismo no nos compromete con un relativismo destructivo ${ }^{3}$. Lo importante es que desde esta perspectiva puede verse cómo estudiar la historia de la ciencia va a contribuir a formar mejores cientificos. Científicos que no serían mejores simplemente porque pueden escribir más artículos que se publican en revistas internacionales o generar mas patentes, sino que serían mejores porque estarlan mejor capacitados para reconocer las posibilidades de la ciencia para criticar o modificar las instituciones de su sociedad.

Hay muchas formas en las que esta idea puede elaborarse, pero lo que si es claro es que el desarrollo de la historia de la ciencia en la segunda mitad del siglo XX no deja lugar a dudas que este tipo de razón es atendible. No es posible desligar tan tajantemente como muchas veces lo requieren los programas de estudio en nuestro medio cuestiones morales, filosóficas e históricas de cuestiones disciplinarias. Una visión más amplia de la historia de una disciplina, y de la manera como esa historia encaja en ámbitos culturales más amplios no sólo fomenta el espíritu crítico e inquisidor de los estudiantes, sino que los ayuda a situar la disciplina en la que quieren especializarse en el contexto de fines y valores de amplio espectro.

El segundo tipo de razón agrega que entender la naturaleza y la estructura de los métodos cientificos requiere entender la historia de esos métodos. Esta afirmación se basa sobre todo en medio siglo de estudio a fondo de la historia de la ciencia que muestra que contrario a lo que sugiere la historia de los libros

3 Estos temas los trato en Filosofla de las Prdcticas Cientificas, en prensa. 
de texto, la formulación de los objetivos y valores que guían la investigación así como la búsqueda de aplicaciones, y sobre todo la interrelación entre métodos, objetivos, valores y aplicaciones no puede entenderse a fondo sin entender la historia de las tradiciones científicas en las cuales esa interrelación entre métodos, objetivos y valores toma forma

Otto Neurath sugiere a principios del siglo XX una idea que sigue siendo pertinente y que en cierto sentido este trabajo elabora en una dirección un poco diferente a la que Neurath sugiere. Para Neurath la elaboración de teorfas no debe verse como una actividad separada de la práctica. Es sólo en un contexto de prácticas en el que podemos darle sentido y entender como conocimiento a una teoría. Las teorías no existen en ningún paraíso platónico o estructuralista, mas bien deben de verse como construcciones intelectuales apoyadas en un contexto de prácticas que se estructuran en tradiciones intelectuales que tienen una historia. Para Neurath era muy importante, y guió mucho de su trabajo profesional, la idea de que esta pretendida preeminencia de la teoŕa en la caracterización de la actividad científica era reforzada por el sistema educativo imperante, y de que cambiar las cosas requería cambiar la manera de enseñar lo que era la ciencia y el papel que la ciencia podía jugar en un proyecto de reconstrucción social. La educación tenia que hacer énfasis en reconocer nuestras limitaciones para proceder racionalmente y en este reconocimiento la ciencia y la enseñanza de la ciencia y de su bistoria tenian que jugar un papel central. Para él, Los frutos de cualquier filosofía que pretendiera servir a un proyecto de emancipación de la sociedad humana tenía pues que pasar por una reforma educativa.

Al tercer tipo de razón subyace una idea muy similar a la del segundo tipo. La historia de la ciencia nos ayuda a ver la manera como desarrollos en diferentes áreas de la ciencia han sido mutuamente interdependientes. Nos ayuda a ver también la manera cómo desarrollos científicos dependen de avances tecnológicos, algo que muchas veces se menciona de pasada pero que no es un tema importante en los libros de texto. Un estudio de la historia de la ciencia puede ayudarnos a superar en buena medida la estrechez del conocimiento que presentan los libros de texto y sobre todo puede ayudar a fomentar una visión humanista de los estudios científicos.

Finalmente, el cuarto tipo de razones depende de supuestos sicológicos y sociológicos. Se asume que la presentación histórica del material no sólo nos ayuda a situar los conceptos de una disciplina como parte de un panorama mas 
amplio, sino que esa presentación es particularmente apropiada para que tenga lugar un entendimiento apropiado del material que desarrollan los libros de texto y las técnicas de laboratorio. Muchos de los grandes promotores de la historia de la ciencia en la educación de la ciencia han sostenido alguna versión de esta idea. Se asume que el desarrollo de la cognición individual de alguna manera puede apoyarse en el desarrollo del entendimiento que tiene lugar históricamente en las comunidades científicas. Esta idea del paralelismo de la cognición individual y la cognición social fue muy extendida en el siglo XIX como parte de una concepción cósmica de la evolución, esto es, como parte de la idea de que todo en el mundo tendía a desarrollarse en una cierta dirección convergente que era el resultado de una ley general del progreso. Esta fue la base del entusiasmo mostrado por algunos positivistas clásicos (como Spencer y Mach) por el estudio de la historia de la ciencia y por su importancia en la educación. Un entusiasmo que cómo mencionamos anteriormente ciertamente no es compartido por muchos positivistas del siglo XX. A principios del siglo XX se sugirieron varias versiones del principio de paralelismo de alcance mucho mas restringido. Muchos libros de texto sugieren la importancia de algún tipo de paralelismo entre el desarrollo individual y el desarrollo de la ciencia aunque no la utilizan por lo general como recurso pedagógico. En el siglo $\mathrm{XX}$ una versión todavía mas restringida de esta tesis, que va acompañada de un programa experimental, es el proyecto de Piaget. Según él hay un paralelismo entre el progreso en la organización lógica y racional del conocimiento y los correspondientes procesos sicológicos formativos en los individuos humanos. Sobre todo las teorías de Piaget han sido muy importantes en el desarrollo de proyectos especificos que intentan relacionar la historia de la ciencia con la educación de la ciencia asumiendo este tipo de paralelismo (referencia).

Por supuesto que todas estas razones pueden ser, han sido, y siguen siendo cuestionadas. Sobre todo el supuesto paralelismo Piagetiano ha sido cuestionado desde la perspectiva de recientes teorías de la cognición humana. Si bien es indudable que cierto paralelismo existe y es explotable en la educación de la ciencia, el paralelismo no tiene porqué darse como Piaget sugiere. En todo caso, estos son temas de discusión entre especialistas que no disminuyen la fuerza de las razones anteriores, simplemente dejan abierta la manera como pueden explotarse en programas de estudio y proyectos educativos a largo plazo.

III. Todas estas razones por lo general se entienden como apoyando el estudio de la ciencia teórica, pero cómo ya lo hemos mencionado hablando de 
Neurath, no tienen porqué verse de esa manera; es más, una vez que la ciencia se piensa como un complejo entramado de prácticas que a su vez se agrupan en tradiciones, estas razones pueden verse como apuntando más claramente al reconocimiento de la ciencia como parte de la cultura. De manera muy breve, una tradición científica para nuestros propósitos genera una manera relativamente estable de plantearse y resolver problemas. Una tradición sería una comunidad de científicos históricamente identificable, por lo general institucionalmente organizados, que cultivan la interacción entre «estilos de pensamiento" y "estilos de hacer las cosas" que se articulan y transmiten a través de prácticas científicas. Una práctica es la concretización de un estilo de pensamiento ylo un estilo de hacer que lleva aparejado criterios (normas implícitas o explícitas) acerca de cuales son las maneras correctas de hacer algo. Por ejemplo, en un laboratorio una práctica científica es el ámbito en el que diferentes tipos de conocimiento especializado (que incluye tipos de saber cómo, habilidades por ejemplo) y de tecnologías se intersectan y generan una cierta operación o proceso distintivo de la práctica. Una práctica taxonómica consiste en una serie de reglas y criterios que llevan a un experto a distinguir dos individuos como miembros de dos especies diferentes, por ejemplo. Toda práctica tiene una base material de cosas hechas de diferentes materiales con propiedades distintivas que constituyen los recursos materiales de la práctica. Las prácticas permiten la comparación de resultados numéricos obtenidos por aparatos, la distinción entre dos tipos de fenómenos, la individuación de un tipo de proceso, etc. Hay diferentes maneras en las que el concepto de práctica puede elaborarse mas a fondo. Andrew Pickering por ejemplo ha caracterizado prácticas en término de actividad material (construcción de aparatos, afinación o calibración de instrumentos) modelos instrumentales (que permiten evaluar los instrumentos existentes y diseñar alternativas) y modelos fenomenológicos (que nos describen las posibilidades de los fenómenos experimentalmente construldos) ${ }^{4}$. Hay otras maneras de hacerlo. Hacking elabora una lista de elementos que juegan un papel en el laboratorio experimental que puede verse como orra manera de caracterizar una práctica de laboratorio. Hacking incluye en su lista cosas tales como las preguntas que se hacen los investigadores, el conocimiento del que parten, las teorías que juegan un papel en el diseño de los experimentos, las hipótesis acerca del tema, los modelos teóricos de los aparatos que

4 "Living in the material world", In Gooding D, T. PINCH, and S. SCHAFFER, comps. The Uses of Experiment: Studies in the Natural Sciences. Cambridge 1989. 
dan cuenta de cómo se entiende el funcionamiento de los aparatos en término de teorías aceptadas ampliamente en la comunidad, y los materiales que entran en el experimento ${ }^{5}$. Yo he hecho énfasis en la importancia que tiene reconocer que el desarrollo de la actividad experimental a través de prácticas de laboratorio puede modelarse como un proceso evolucionista, lo que permite entender la manera como las normas implícitas en prácticas se transmiten y extienden o modifican de una práctica a otra, lo que a su vez permite explicar el origen de por lo menos algunas normas epistémicas ${ }^{6}$. No es este el lugar para elaborar el concepto de práctica en sus diferentes versiones, ni el lugar de hacer ver la importancia que tienen en los estudios contemporáneos sobre la ciencia. Aquí es suficiente con que el lector tenga una idea intuitiva de práctica en el sentido que es pertinente para hacer el contraste entre filosofia de la ciencia teórica (la filosofía de la ciencia que considera que en última instancia lo filosóficamente interesante de la ciencia es la estructura y la evaluación de teorías, y la filosofía de la ciencia que reconoce la importancia de las prácticas científicas y los "estilos de hacer" (tecnología) en la construcción y evaluación del conocimiento cientifico.

Pasemos ahora a reseñar muy brevemente algunas de las razones que pueden darse a favor de enseñar la historia de la ciencia en general, tanto en la escuela secundaria como en la universidad. Las primeras dos razones anteriores son también razones que pueden esgrimirse en el contexto educativo amplio. Partimos del supuesto que la educación debe contribuir al estudio de los aspectos culturales más relevantes para poder entender el tipo de sociedad en la que vivimos. En la medida que la ciencia es un complejo de recursos culturales que juegan un papel importante en la dinámica social el estudio de la ciencia es deseable no sólo para los estudiantes de ciencias sino para todos los ciudadanos. Una versión de la tercera razón mencionada anteriormente, que puede utilizarse para argumentar por la ensenanza de la historia de la ciencia en general es la siguiente: el estudio de la historia de la ciencia y la tecnología fomenta el entendimiento de la manera como el mundo social se ha desarrollado y constituido, y por lo tanto contribuye a lograr uno de los objetivos centrales de cualquier proyecto educativo, proveer conocimiento que sitúe a

5 "The Self-Vindication of the Laboratory Sciences", in Science, Practice and Culture, compilado por A. PICKERING, Chicago 1992.

6 "La Autonomía de las tradiciones cientificas", el caso de las tradiciones experimentales", Critica 80, agosto de 1995. Ver también Filosofla de las Prácticas científicas, UNAM, en prensa. 
las personas en un ámbito que les permita contribuir al bienestar propio y colectivo.

Paso ahora a mostrar cómo diferentes concepciones de lo que se entiende por conocimiento tienen diferente alcance con respecto a este objetivo. En particular, quiero mostrar que si se abandona la concepción tradicional según la cual el conocimiento científico consiste de teorías, entonces puede argumentarse de manera mas convincente y mas integrada que el estudio de la historia de la ciencia y la tecnología contribuye a lograr el objetivo anteriormente mencionado.

Según la concepción tradicional son las teorías lo que guía a la razón en conflictos entre creencias y en decisiones prácticas. La educación de la historia de la ciencia desde esta perspectiva sólo puede ser un sucedáneo para aquellos que no han estudiado ciencia teórica. Se considera que las teorías cientificas nos dan una visión "desde ninguna parte» y por lo tanto objetiva del conocimiento. De aquí es fácil pasar a la tesis que el conocimiento de la teoría es el fin de la ciencia, y por lo tanto cualquier otra manera de conocer la ciencia es secundario. El conocimiento de la historia de la ciencia puede ser una motivación adicional para estudiar la ciencia, servir de depositario de ideales de conducta para aspirantes a científicos por ejemplo, o servir como un medio para divulgar la ciencia a aquellos que no tienen la capacidad para estudiarla profesionalmente, pero no puede servir para mucho mas que eso.

Consecuentes con esta concepción del conocimiento varios historiadores han sugerido que el estudio de la historia de la ciencia debe de dejarse a especialistas, e incluso, que los estudiantes de ciencia en particular no deberían estudiar historia de la ciencia. Thomas Kuhn, por ejemplo, arguye que puesto que el entrenamiento riguroso de los científicos requiere de un cierto tipo de dogmatismo que aleje a los estudiantes de maneras alternativas de enfocar los problemas, el estudio de la historia de la ciencia sería una distracción poco formativa, o incluso podría ser una distracción nociva ${ }^{7}$. Otros críticos del uso de la historia de la ciencia como un recurso pedagógico plantean una objeción relacionada con la anterior. Martín Klein, un renombrado historiador de la física, sugiere en un artículo publicado en 1972 que el uso selectivo y con fines pedagógicos de material histórico "corre el riesgo de cometer una injusticia a

7 KUHN T. "The essential Tension: Tradition and Innovation in Scientific Research", in The Essential Tension, Chicago 1977. El artículo fue publicado originalmente en 1959. 
la fisica o a su historia" ${ }^{8}$. Muchos historiadores de mediados del siglo XX han argüido que puesto que la historia de la ciencia debe de estudiarse esencialmente en el contexto intelectual en el que surgió, el utilizar la historia de la ciencia con fines pedagógicos para visualizar la estructura de la ciencia contemporánea no puede sino resultar en un mal uso de la historia ${ }^{9}$. Se asume que hay una división de tareas importante. Mientras que los historiadores se preocupan por hilvanar los detalles de un suceso en una narrativa que busca poner en perspectiva la complejidad del pasado, los científicos deben de evitar en lo posible las narrativas y atenerse a los hechos.

Nótese que en el fondo detrás de este tipo de objeciones a la enseñanza de la historia de la ciencia está el presupuesto de que el conocimiento es conocimiento de teorías. La objeción tiene sentido sólo si se asume que la ciencia es algo como lo que Kuhn llama ciencia normal, una actividad dirigida y orientada por una cierta teoría (junto con (o a través de) aplicaciones ejemplares). Si por ejemplo Kuhn pensara que el estudio de la historia de la ciencia puede ayudarnos a entender la manera como los diferentes tipos de recursos se organizan en prácticas científicas, y que saber acerca de esto es parte del saber cientifico, entonces no tendría sentido su objeción. Tanto los aprendices de científico como cualquier ciudadano pueden sacar partido de conocer la estructura de los recursos que han guiado la construcción de una empresa tan compleja como la ciencia.

La segunda objeción igualmente asume que es posible atenerse a los hechos y a las teorías que les sirven de marco explicativo, y que por lo tanto cualquier narrativa que no tiene como base ese marco teórico-explicativo no puede ser sino algo no sólo dispensable sino potencialmente engañoso.

Cursos sobre diferentes tipos de estudio sobre la ciencia, por ejemplo de "Ciencia, Tecnología y Sociedad", de "Ciencia y Género", de "Ciencia y política", están mostrando cada vez de manera mas clara que los estudios empíricos sobre la ciencia, y la historia de la ciencia en particular, pueden desempeñar un papel importante en educar a los ciudadanos respecto a la naturaleza de la ciencia y su importancia para entender y criticar maneras específicas de planear el

8 Cita tomada del artículo de Matthews mencionado en la nota 2.

9 "Use and abuse of Historical Teaching in Physics", in S.G. BRUSH, y A.L. KING compiladores, History in the Teaching of Physics, University Press of New England, Hanover, NH. Estos y otros argumentos en contra del estudio de la historia de la ciencia se presentan en el artículo de Matthews mencionado en la nota 2. 
desarrollo social. Temas como la contaminación ambiental, el papel de los científicos como expertos, la promoción y evaluación de fuentes alternativas de energía, políticas de preservación o restauración de medio ambientes etc. requieren precisamente educar a los estudiantes en el tipo de argumentación polifónica que historiadores como Klein piensan que no es propio de la ciencia, ya que piensan la ciencia como eminentemente conocimiento teórico.

Si pensamos el conocimiento científico como todo el complejo de instituciones y prácticas que constituyen recursos para la indagación racional entonces las objeciones anteriores a la enseñanza de la historia de la ciencia desaparecen. Es mas, las razones que usualmente se dan para enseñar historia de la ciencia, algunas de las cuales hemos comentado en páginas anteriores, adquieren mayor realce. Estas razones son mutatis mutandis razones para estudiar la ciencia desde perspectivas de crítica social, a través de cursos sobre cuestiones éticas y de género, o sobre cuestiones conflictivas que plantea el desarrollo de la tecnología.

En primer lugar es importante dejar claro que mi propuesta no reduce el conocimiento científico a un mero sistema de creencias o habilidades. Las creencias y habilidades que forman parte de una práctica están configuradas alrededor de objetivos que por lo menos muchas veces (o indirectamente) son epistémicos. Estos objetivos toman cuerpo a través de normas implícitas o explícitas en esas prácticas que nos dicen que es correcto o incorrecto en por lo menos ciertas situaciones epistémicas. Las normas implícitas en prácticas pueden verse como lineamientos para la construcción de situaciones epistémicas, esto es, de situaciones en las cuales podemos decir que la práctica se ejecuta correctamente desde una perspectiva epistémica, por ejemplo, desde una perspectiva donde se considera que se avanza el conocimiento. En un laboratorio el diseño y preparación de un experimento es la preparación de una situación en la cual podemos utilizar las normas (en relación con los objetivos) para decidir si algo es o no el caso. Lo que es importante recalcar es que tenemos confianza en que las normas de la práctica van a generarnos un resultado confiable porque hemos construido la situación de manera tal que ese resultado sea confiable. Este tipo de construcción del resultado es similar al proceso por medio del cual se llega a caracterizar una ley de la naturaleza ${ }^{10}$. Es también similar al procedimiento por medio del cual se justifican

10 Ver el libro de Nancy Cartwright The Dappled World, 1999. 
y modifican a través de la historia de la ciencia los patrones de explicación causal ${ }^{11}$.

Es claro que entender esta estrategia de lo que podemos llamar equilibrio reflexivo requiere estudiar casos concretos en los cuales la estrategia ha sido efectiva ${ }^{12}$. No hay un algoritmo o método general que nos permita decidir sobre el alcance de las normas o acerca de cual es la taxonomía de los fenómenos adecuada para la aplicación de las normas. Estudios concretos de este tipo de estrategia reflexiva (en diferentes tipos de indagaciones racionales) es crucial para que la educación cumpla el objetivo de situarnos criticamente en una historia y una sociedad particular. Es claro que desde esta perspectiva cualquier argumento a favor de estudiar la historia de la ciencia es un argumento a favor de incorporar en el currículo el estudio crítico de la ciencia desde las diversas perspectivas que puedan ser importantes socialmente en una comunidad determinada. El tema del cientifico como experto social es uno de estos temas en donde de manera natural la historia de un problema desempeña un papel importante en la evaluación del conocimiento como en la terapia que puede sugerirse. Este tipo de discusiones permite que los estudiantes entiendan la manera como la normatividad científica depende de una discusión sobre el alcance de las normas sin que sea necesario que entiendan las sutilezas de la formulación del problema desde una perspectiva disciplinar. Algo que por ejemplo es más difficil de lograr si se estudia la historia de las ciencias de laboratorio con estudiantes sin la preparación cientifica correspondiente. Además, este tipo de discusiones permiten claramente ver porqué es importante que los cientificos tengan una preparación que incluya el estudio de la ciencia desde una perspectiva humanista, desde el tipo de perspectiva polifónica que para autores como Klein y Kuhn, y muchos cientificos a cargo de organizar los currículos, corre el riesgo de desencarrilar a los estudiantes. Veamos un ejemplo.

V. A principios de los años ochenta en una pequeña comunidad en Aspen Colorado (en E.E.U.U.) se descubrió que el suelo estaba fuertemente contaminado por plomo. La agencia gubernamental que supervisa los problemas del

11 Martinez Sergio, De los Efectos a las Causas, Paidos-UNAM, México 1997.

12 Requeriría un poco de argumento mostrar el sentido en el que este tipo de estrategia es en cierto sentido análoga al equilibrio reflexivo inicialmente propuesto por Nelson Goodman (para una justificación de la inducción) y a la estrategia de John Rawls en ética (quién introduce la terminologia). Esto no va a hacerse aquí. 
medio ambiente en ese país, la agencia para la protección del medio ambiente, la EPA (por sus siglas en inglés), llevó a cabo una serie de estudios y en 1986 propuso que debería de removerse mas de un metro de suelo en toda el área residencial y cambiarla por un suelo no contaminado. La EPA hizo una reunión y notificó a los residentes de su decisión. Los residentes empezaron a movilizarse y a pedir estudios por parte de otras instituciones y llegaron a la conclusión que la EPA no parecía tener evidencia sustancial de que hubiera algún impacto negativo en la comunidad por la presencia de plomo en el suelo. Generalmente se asume que sobre todo los niños menores de 6 años están muy propensos a contaminarse porque juegan en el suelo y no se lavan las manos muy seguido. Lo sorprendente del estudio promovido por la comunidad fue que si bien el suelo estaba contaminado los niveles de plomo en la sangre de los residentes, incluyendo los niños, estaban incluso muy por debajo de la media nacional.

No obstante la EPA seguía queriendo imponer lo que la agencia consideraba era la medida mas apropiada para promover la buena salud de los ciudadanos, remover cerca de un metro de suelo. Finalmente se acordó hacer un estudio independiente por parte de un comité de expertos que sería acordado por las partes, tanto la EPA como los residentes tenían derecho de vetar a alguien sin tener que dar razones. El comité técnico finalmente seleccionado tenía la tarea de revisar los documentos existentes acerca del nivel de plomo en el suelo, el impacto en la salud de los habitantes, recabar y evaluar el testimonio de residentes de Aspen y de la EPA para finalmente llegar a una decisión.

La tesis defendida por EPA era que el plomo estaba en el suelo y que eso era un riesgo significativo, si había o no causado daño hasta ahora eso no era importante. Los residentes sostenían que mientras no vieran claro que podía causar daño no procedía la remoción del suelo. El comité de técnicos corroboró que los estudios que permitian inferir que la población tenía un bajo nivel de plomo en la sangre era en efecto representativos de la población, y varios expertos trataron de mostrar que esto se debla a que la manera como el plomo se presentaba en el suelo era poco asimilable. Los miembros del comité no consideraban totalmente convincente este tipo de argumento, ni muchos otros que se presentaron, tomándolos uno a uno, pero si consideraron convincente el hecho que todos estos argumentos apuntaban en la misma dirección para el caso concreto que estaba en consideración. Hay estudios que muestran que los niños bien nutridos tienen menos riesgo de contaminarse con plomo proveniente del suelo. Otros estudios sugieren que cuando el suelo está predominantemente cubierto por pasto u otro tipo de vegetación el riesgo de contaminación por plomo disminuye. Todos 
estos y otros factores permitían explicar los bajos niveles de plomo en la sangre y permitian predecir que para esa comunidad el riesgo de que la situación cambiara era despreciable. Se concluyó que si bien había una mínima probabilidad de que en el futuro el plomo en el suelo se constituyera en una amenaza para la salud de la comunidad esta probabilidad era despreciable. La comunidad en cuestión era tal que todas las condiciones requeridas para que la asimilación del plomo del suelo no fuera un problema se cumplían. Por ello llegaron a la conclusión que la remoción del suelo no era necesaria para preservar la salud de la población, y se recomendó tener un programa que evaluara constantemente la situación.

Este es un ejemplo de cómo los científicos pueden jugar un papel importante como expertos sociales en un sentido que claramente permite ayudar a una comunidad a llegar a una decisión respecto a un tema en el que la decisión a tomar requiere de la capacidad para identificar y evaluar riesgos de una manera novedosa, en situaciones en las que no se pueden aplicar reglas simples para tomar decisiones apropiadas. Este no es el tipo de tarea para la que los cientificos están entrenados, pero que cada vez puede ser mas importante que lo estén. Nuevamente, desarrollar esta capacidad de los científicos requeriría no simplemente aprender a resolver problemas propios de la disciplina sino poder integrar una gran diversidad de valores en el planteamiento de los problemas. El estudio de la historia de la ciencia y la tecnología, así como el estudio de problemas éticos que genera el desarrollo y la aplicación de avances científicos ciertamente contribuiría a la tarea. Este tipo de ejemplos muestran claramente la verdad de la afirmación de Neurath de que que cambiar la manera como se toman socialmente las decisiones requiere cambiar la manera de enseñar la ciencia ${ }^{13}$.

Este ejemplo muestra también el estira y afloje que muchas veces caracteriza la discusión acerca del alcance de las normas científicas. Situaciones muy similares son muy comunes en las ciencias experimentales, en donde muchas veces el planteamiento del problema concreto lleva a una discusión acerca de la pertinencia o no de normas que la situación concreta muestra que pueden entrar en conflicto. La decisión acerca de la pertinencia de una norma en estos casos muchas veces depende de una interpretación de la situación que se va

i3 El ejemplo de esta sección proviene de una conferencia presentada en un simposio titulado "How Clean is Clean enough" que tuvo lugar en la universidad de Colorado en Boulder el 6 de octubre de 1993. Puede leerse en http://campuspress.colorado.edu/CEJ/Brfings/Clean.html. 
desarrollando en el proceso de la toma de decisión y que por lo tanto puede ir cambiando dependiendo del alcance que se decida darle a las normas. A través de este proceso normas implícitas en prácticas van delineándose explícitamente, pero muchas veces esta delineación explícita de la norma no llega a ser definitiva o a capturar significativamente la norma implícita. Entender esta dialéctica compleja entre normas implícitas y explícitas es importante para no trivializar el concepto de norma implícita en prácticas. Una norma implícita no es simplemente una norma que en principio puede hacerse explícita. El proceso a través del cual las normas implícitas se hacen explícitas en tipos de argumentación es un proceso que está íntimamente ligado con la búsqueda por plantearse y resolver la pertinencia de diferentes tipos de prácticas para una determinada decisión, que puede ser una decisión acerca de aceptar o no una teoría o acerca de llevar a cabo o no un experimento, o acerca de si una serie de observaciones justifica una determinada conclusión. El proceso de irse haciendo explícita una norma implícita es parte del proceso de ir caracterizando situaciones concretas como de cierto tipo, y por lo tanto es parte del proceso por medio del cual se van individualizando normas en la medida que se va estabilizando lo que se considera es el alcance de las normas. Esto puede ser un proceso muy largo e históricamente complejo.

VI. Resumamos cómo esta manera de entender el conocimiento científico como articulado en prácticas nos permite ver desde una perspectiva muy diferente a la tradicional las razones por las que es importante y serfa deseable que la historia de la ciencia se enseñara, tanto en facultades de ciencia como a otros niveles educativos. En primer lugar es importante hacer ver que desde la perspectiva tradicional el conocimiento científico es muy diferente de otros tipos de conocimiento. El conocimiento moral, estético, de uno mismo o de los demás, es desde la perspectiva tradicional muy diferente del conocimiento científico ya que sólo este último no se entiende como un logro generalizado de los seres humanos. El conocimiento científico es el tipo de conocimiento que tienen los científicos sobre sus temas de especialización. Desde la perspectiva que promovemos el conocimiento científico es un logro generalizado de los seres humanos. Esto no quiere decir que todos sabemos lo que los cientificos saben, sino que la responsabilidad sobre el uso y las direcciones de desarrollo del conocimiento científico-tecnológico es de todos, y que el ejercicio de esa responsabilidad es parte esencial de lo que debemos entender por conocimiento cientifico y por lo tanto debe de ser parte esencial de la educación generalizada. Las prácticas científicas no son transparentes a los científicos y opacos a los demas. 
Las prácticas científicas, como lo ilustra el ejemplo anterior de la controversia sobre la contaminación del suelo de Aspen, no son ni transparentes ni opacas para un grupo determinado. Las prácticas científicas, y mas especificamente las normas implícitas en ellas, se vuelven transparentes (en el sentido que su alcance queda delimitado en una situación particular) a lo largo de un complejo proceso de evaluación de la evidencia disponible. Una vez que se acepta que el conocimiento científico es un conocimiento generalizado las preocupaciones reseñadas anteriormente respecto a la diferencia entre la historia y la física, o entre el tipo de educación que debe de recibir un cientifico y el tipo de conocimiento que promueve la historia dejan de ser preocupantes.

Un argumento que muchas veces ha sido utilizado implícitamente en discusiones sobre el tema es que la educación secundaria, como parte de la educación generalizada de todos los ciudadanos, debe de promover todo aquello que sea conocimiento generalizado. Si alguien quiere estudiar música o ciencia o historia de la ciencia eso está muy bien pero este tipo de temas no deben de ser parte del currículo general. A este argumento puede respondérsele como sigue. Como hemos visto entender lo que es y lo que no es conocimiento está íntimamente ligado al problema de entender el alcance de las normas científicas, y sobre todo de las normas implícitas en prácticas. En la medida que tanto el estudio de la música como el estudio de la ciencia pueden ayudar a ejercitarnos en la difícil tarea de interpretar normas, y particular determinar su alcance, el estudio de la historia de la ciencia como el de la música es recomendable. A diferencia de la música, sin embargo, el estudio de la ciencia puede ayudarnos a mejorar nuestra manera de llegar a decisiones colectivas racionales, y por lo tanto puede contribuir significativamente a un desarrollo de la ciencia y la tecnología genuinamente guiado por valores que promuevan el florecimiento generalizado de los seres humanos. Desde esta perspectiva es claro que un objetivo de la educación debe de ser promover el estudio de la ciencia como un conocimiento generalizado, de la misma manera que la educación debe de promover el conocimiento de uno mismo o de los demás.

Un curso de la historia de la ciencia no debe de restringirse a un estudio de la historia de cómo la indagación racional ha llevado a la propuesta y aceptación de teorías. La historia de la ciencia es mas pertinente como parte de un proyecto de educación generalizada en la medida que nos permite vislumbrar de manera concreta la manera como un proceso de decisión puede tomar en cuenta el juicio de expertos en cuestiones que involucran valores y riesgos, y cosas que no sabemos, que no pueden ser resueltos por una simple aplicación de reglas o de manuales. 